\title{
Determinação do volume de anestésico local para bloqueio espinal toracolombar (T13-L3), em cães com peso entre 20 e $40 \mathrm{~kg}$
}

\author{
[Determination of the local anesthetic volume for toracolumbar (T13-L3) spinal block in dogs \\ between 20 and $40 \mathrm{~kg}$ ]
}

\section{"Comunicação Breve/Short Communication"}

\author{
Kassia Fernanda Araujo Damasceno ${ }^{*}$, Maria Raquel Almeida, Jacinta Eufrasia Brito Leite, \\ Eduardo Alberto Tudury
}

Departamento de Medicina Veterinária, Universidade Federal Rural de Pernambuco, Recife-PE, Brasil. *Autor para correspondência/Corresponding author: E-mail: kassiafernadaad@hotmail.com

\section{Resumo}

Já é reconhecido que os bloqueios nervosos com anestésicos locais são capazes de aliviar a sensação dolorosa severa, possibilitando ainda no trans-operatório uma anestesia geral mais superficial, com menores transtornos cardiovasculares e respiratórios, diminuindo assim os riscos anestésicos. O bloqueio epidural toracolombar, por ser um tipo de anestesia regional, pode cumprir estes requisitos ante à dor gerada por cirurgias espinais toracolombares, comum em cães com doença do disco intervertebral (DDIV). Este trabalho experimental utilizando cadáveres, teve como objetivo verificar o volume necessário de anestésico local para viabilizar a realização de bloqueio espinal toracolombar de $\mathrm{T}_{13}$ até $\mathrm{L}_{3}$ em cães com peso entre 20 e $40 \mathrm{~kg}$, que necessitassem de neurocirurgias descompressivas por DDIV. Foram utilizados 10 cadáveres de cães com este intervalo de peso, sem histórico de afecção espinal, onde se mediu o comprimento em centímetros, da coluna vertebral $\mathrm{T}_{13}-\mathrm{L}_{3}$. Foi colocado um cateter no espaço epidural pelo forame vertebral de $\mathrm{L}_{5}, \mathrm{~L}_{6}$ ou $\mathrm{L}_{7}$ até à vértebra $\mathrm{L}_{3}$ e realizada secção vertebral entre $\mathrm{T}_{12}-\mathrm{T}_{13}$ para visualização do forame vertebral de $\mathrm{T}_{13}$ e verificação do volume de solução de corante Giemsa necessário para que o mesmo chegasse nesse local, quando injetado pelo cateter com sua ponta situada exatamente ca u dal à $L_{3}$. Posteriormente foi injetada a solução diluída de contraste de bário e se verificou (pela extensão do contraste na radiografia) que o volume determinado com o corante Giemsa foi suficiente para o preenchimento epidural da junção toracolombar a ser submetida à descompressão medular, caso o animal tivesse uma protrusão discal. Com esses resultados foram calculadas médias e desvio padrão do volume por cm de coluna vertebral entre $\mathrm{T}_{13}$ e $\mathrm{L}_{3}$. O volume de solução de anestésico que deveria se aplicar por cm de coluna vertebral entre $\mathrm{T}_{13}$ e $\mathrm{L}_{3}$, de cães com peso entre 20 e $40 \mathrm{Kg}$, oscilaria entre 0,059 e $0,109 \mathrm{ml}$, com média e desvio padrão de $0,084 \pm 0,01683 \mathrm{ml}$.

Palavras-chave: caninos; coluna vertebral; anestesia; cirurgia espinal.

\begin{abstract}
It is already recognized that nerve blocks with local anesthetics are able to alleviate severe pain sensation, allowing a more superficial general anesthesia, with minor cardiovascular and respiratory disorders, reducing anesthetic risks. Thoracolumbar epidural block, as a type of regional anesthesia, could meet these requirements in cases of pain generated by thoracolumbar spinal surgeries, common in dogs with intervertebral disc disease (DDIV). The objective of this study was to verify the amount of local anesthetic necessary for $\mathrm{T}_{13}-\mathrm{L}_{3}$ epidural anesthesia in dogs weighing between 20 and $40 \mathrm{~kg}$, that required decompressive neurosurgery due to DDIV. Ten cadavers of dogs weighing between 20 and $40 \mathrm{~kg}$ were used, with no history of spinal affection, where the length in centimeters of $\mathrm{T}_{13}-\mathrm{L}_{3}$ vertebral column was measured. A catheter was placed in the epidural space through the vertebral foramina of $\mathrm{L}_{5}, \mathrm{~L}_{6}$ or $\mathrm{L}_{7}$ up to $\mathrm{L}_{3}$ vertebra. Subsequently, a vertebral section was performed between $T_{12}-T_{13}$ to visualize $T_{13}$ vertebral foramen and verify the volume of Giemsa dye solution needed for it to arrive at that site, when injected by the catheter located at a flow rate of $\mathrm{L}_{3}$. In the segments of the vertebral column collected and preserved from the 10 individuals, diluted barium contrast solution was subsequently injected. At this time, it was verified by the extent of contrast on the radiograph, that the volume
\end{abstract}


determined in the first part of the experiment was sufficient for epidural filling of the thoracolumbar junction intended to undergo spinal decompression if the animal had a disc protrusion. With these results, mean and standard deviation of the volume per $\mathrm{cm}$ of vertebral column between T13 and L3 were calculated. The volume of anesthetic solution to be applied per cm of vertebral column between T13 and L3 would range from 0.059 to $0.109 \mathrm{~mL}$, with mean and standard deviation of $0.084 \pm 0.01683 \mathrm{~mL}$.

Keywords: canine; spine; anesthesia; spinal surgery.

A anestesia epidural, empregada desde o início do século XX (Franco e Diz, 2000), é indicada em procedimentos obstétricos, ortopédicos dos membros posteriores e no controle de dor pós-operatória (Carvalho e Luna, 2007), e tem como vantagens o emprego de doses menores de fármacos e anestésicos gerais, poucos efeitos colaterais, além de proporcionar planos anestésico mais superficiais (Massone, 2003; Futema, 2010; Otero, 2013; Skarda e Tranquilli, 2013). O bloqueio epidural torácico é pouco utilizado em neurocirurgia animal, apesar de ter como característica a prevenção de hipóxia e melhora na atividade respiratória devido à analgesia mais intensa (Vilela e Tudury, 2009).

As características dos bloqueios epidurais devem ser levadas em consideração para 0 cálculo da dose para o paciente, além de outros parâmetros, como o peso corporal do animal e o comprimento da coluna vertebral (Freire, 2011; Otero, 2013). O uso do comprimento da coluna vertebral se baseia no valor em centímetros medidos desde o osso do occipital até a primeira vértebra coccígea (Lumb e Jones, 1973; Almeida et al., 2007; Otero, 2013, Otero et al., 2018). Sabendo que o volume a ser administrado desempenha um importante papel na extensão do bloqueio nervoso, esta medida deve ser bem aplicada, pois na espécie canina existem diferenças no comprimento do canal vertebral (Jones, 2001; Collier, 2010; Villela, 2012; Otero, 2013, Otero et al., 2018). O uso de grandes volumes na anestesia epidural pode comprometer a função dos músculos responsáveis pela ventilação do paciente, assim como a manutenção da pressão arterial dentro de parâmetros de normalidade (Otero, 2013).

$\mathrm{O}$ volume a ser injetado de anestésico local, através de cateter introduzido no espaço $\mathrm{L}_{7}-\mathrm{S}_{1}$, para se abranger o espaço epidural entre $T_{9}-L_{1}$, em cães sem raça definida, com peso entre 10 a $15 \mathrm{~kg}$, deve ser de $0,042 \mathrm{~mL}$ por $\mathrm{cm}$ do comprimento da coluna vertebral toracolombar, em cães com comprimento da coluna $\left(\mathrm{L}_{1}-\mathrm{T}_{9}\right)$ variando de $7,8-$ 12,3cm (Fernandes, 2016). Otero et al. (2018) preconizam que injetando a solução anestésica a partir do espaço lombossacro para bloqueio até $\mathrm{L}_{1}$, o volume deve ser de $0,05 \mathrm{~mL} / \mathrm{cm}$ de coluna, e quando for necessário o bloqueio até $\mathrm{T}_{9}$, o uso de $0,10 \mathrm{~mL} / \mathrm{cm}$ de coluna seria o recomendado. Isto concorda com o descrito por Lumb e Jones (1973), quando estes indicam para anestesia epidural em cães, cuja distância entre a crista occipital e a base da cauda é de $40 \mathrm{~cm}$, que seja usado $0,05 \mathrm{~mL} / \mathrm{cm}$. Entretanto, estes mesmos autores sugerem que para cães de grande porte seja administrada de 0,075 a $0,1 \mathrm{~mL} / \mathrm{cm}$.

A compressão da medula espinal ocorre em cães devido a causas endógenas ou exógenas, como protrusão discal. As lesões se localizam frequentemente na região toracolombar (Brisson, 2010). A utilização de bloqueio epidural pode ser usada em cirurgias descompressivas de pacientes com essa afecção, a qual exibe grande incidência dessa afeção na clínica de pequenos animais (Fernandes, 2016).

A doença do disco intervertebral (DDIV) é causa comum de déficits neurológicos nos cães. Ocorre também em cães de grande porte, exibindo, no entanto, uma menor consequência de prejuízo neurológico por esta enfermidade que os condrodistróficos, visto que apresentam maior espaço epidural quando comparado aos cães toys (Lecouter e Child, 1997; Brisson, 2010; Marioni-Henry, 2010). Na síndrome toracolombar, os cães de peso superior a $20 \mathrm{~kg}$ têm como principais discos afetados os situados entre $T_{13^{-}}$ $\mathrm{L}_{3}$, enquanto que nos condrodistróficos estes situam-se entre $\mathrm{T}_{9}$ e $\mathrm{L}_{1}$ (Brisson, 2010).

A indicação geral para descompressão medular é cirúrgica, por meio da remoção de parte das vértebras e/ou da massa invasora do canal vertebral (Coates et al., 2007). Apesar de serem procedimentos rápidos, são geradores de dor severa (Brisson, 2010), recomendando-se, portanto, procedimentos anestésicos trans e pósoperatórios com eficiente controle da dor (Carvalho e Luna, 2007). 
O objetivo desta investigação foi verificar o volume necessário de anestésico local para viabilizar o preenchimento do espaço epidural $\mathrm{T}_{13^{-}}$ $\mathrm{L}_{3}$, injetado através de cateter epidural lombossacral, para bloqueio espinal toracolombar desse segmento, em cães com peso entre $20 \mathrm{e} 40 \mathrm{~kg}$, que necessitem de neurocirurgias descompressivas por DDIV.

A pesquisa foi desenvolvida no Laboratório de Práticas Post Mortem e no setor de radiologia do Departamento de Medicina Veterinária da Universidade Federal Rural de Pernambuco (UFRPE), sob autorização da CEUA de número 0001/2014.

Foram utilizados 10 cadáveres de cães com peso entre 20 e $40 \mathrm{~kg}$, sem histórico de afecção espinal, fornecidos pelo Centro de Vigilância Ambiental da prefeitura da cidade do Recife ou que vieram a óbito no Hospital Veterinário da UFRPE ou clínicas particulares de Recife, com consentimento dos seus tutores.

Para tal, nos animais foi colhido $o$ segmento vertebral entre $\mathrm{L}_{5}, \mathrm{~L}_{6}$ ou $\mathrm{L}_{7}$ (a depender das dificuldades encontradas na desarticulação) e $T_{13}$. Nos 10 cadáveres, se mediu o comprimento em centímetros (com régua metálica), da coluna vertebral $\mathrm{T}_{13}-\mathrm{L}_{3}$, palpando-se as porções cranial e caudal dos processos espinhosos vertebrais. Na sequência, foi colocado um cateter na região epidural pelo espaço intervertebral $\mathrm{L}_{5}, \mathrm{~L}_{6}$ ou $\mathrm{L}_{7}$ até o limite caudal da vértebra $\mathrm{L}_{3}$. Posteriormente foi realizada secção vertebral entre $T_{12}-T_{13}$ para visualização do limite cranial do forame vertebral e verificação do volume de líquido (solução de corante azul Giemsa com água) que seria necessário para que o mesmo chegasse nesse local, quando injetado pelo cateter situado em $\mathrm{L}_{3}$.

O comprimento de cateter a ser introduzido e levado até $\mathrm{L}_{3}$ foi determinado descontando-se a distância entre o processo espinhoso dessa vértebra e forame vertebral de $\mathrm{T}_{13}$. Com o cateter, já preenchido com solução de corante e introduzido no espaço epidural lombar até a vértebra $\mathrm{L}_{3}$, injetou-se a solução aquosa de Giemsa, com densidade visivelmente semelhante ao dos anestésicos locais usados em anestesia epidural. Nestes 10 animais foi medido o volume médio por $\mathrm{cm}$ de coluna vertebral que foi necessário para preencher o espaço entre $\mathrm{L}_{3}$ e $\mathrm{T}_{13}$.

Posteriormente nos segmentos vertebrais colhidos dos 10 indivíduos, foi injetado por cateter semelhante, com sua ponta atingindo $\mathrm{L}_{3}$ no espaço epidural do canal vertebral, o meio de contraste solução de sulfato de bário a $100 \%$ $\left(\right.$ Barioge $^{\circledR}{ }^{\circledR}$ ) diluída com 1/4 de água destilada e 3/4 da solução, no mesmo volume que havia sido determinado com o corante Giemsa. Nesse momento se procurou verificar pela extensão do contraste, em radiografias, se o volume determinado na primeira parte do experimento seria suficiente para o bloqueio regional epidural da junção toracolombar pretendida.

As médias e desvios padrões respectivas ao peso, idade, distância $\mathrm{T}_{13}-\mathrm{L}_{3}$ e volume injetado total se encontram na Tabela 1. Considerando-se os segmentos vertebrais de cães com peso entre 20 e $40 \mathrm{~kg}$, o volume de solução de anestésico que deveria se aplicar por $\mathrm{cm}$ de coluna vertebral entre $T_{13}$ e $L_{3}$ oscilaria entre 0,059 e $0,109 \mathrm{~mL}$, com média de $0,084 \pm 0.01683 \mathrm{~mL}$, discordando dos volumes indicados por Otero et al. (2018), que ao pretender anestesiar o espaço epidural de seis vértebras lombares (L7-L1), recomendou usar $0,05 \mathrm{~mL} / \mathrm{cm}$. Nesta pesquisa se calculou o volume epidural de quatro vértebras (L3-T13), ao se administrar a solução com cateter epidural, e foi constatado que o volume a ser administrado por $\mathrm{cm}$ é superior a esse valor. Tal fato reforça a afirmação de Lecouter e Child (1997), Brisson (2010) e Marioni-Henry (2010) de que cães de grande porte têm um espaço epidural maior quando comparado aos de pequeno porte.

Em cães de grande porte, Lumb e Jones (1973) indicam utilizar o volume compreendido entre $0,075-0,1 \mathrm{~mL} / \mathrm{cm}$, corroborando os achados deste trabalho. Sendo que em pesquisa semelhante a esta, para se preencher o forame vertebral de quatro vértebras de cães com peso entre $10 \mathrm{e} 15 \mathrm{~kg}$, só foi necessário utilizar $0,042 \mathrm{~mL} / \mathrm{cm}$ (Fernandes, 2016), pode-se afirmar que quando se pretende realizar anestesia epidural (através de cateter epidural) do segmento vertebral, principalmente quando afetado pela DDIV, o volume por $\mathrm{cm}$ deverá ser distinto, conforme o porte do animal.

$\mathrm{O}$ fato dos valores de volume por $\mathrm{cm}$ espinal não seguirem exatamente uma correspondência quanto ao peso dos animais pode ser devido a não terem todos o mesmo escore corporal, bem como fatores de conformação corporais individuais ou de raça. Acredita que o fato da coluna vertebral estar seccionada, não trouxe influenza no volume necessário de preenchimento epidural, pois o cateter foi colocado com sua ponta em caudal de L3, dirigindo o líquido 
(Giemsa e Bario) para cranial, tendo sempre gordura epidural caudal a esse ponto, atuando como um limitante ao refluxo. Em nenhum caso o produto injetado emergiu no forame vertebral caudal exposto do segmento espinal em estudo.

Tabela 1. Dados das unidades experimentais relativos à raça, peso corporal, idade, comprimento do segmento $\mathrm{T}_{13}-\mathrm{L}_{3}$, volume de corante necessário de preenchimento dessa distância e volume por $\mathrm{cm}$ desse segmento.

\begin{tabular}{|c|c|c|c|c|c|c|}
\hline Cão & Raça & Peso (Kg) & $\begin{array}{l}\text { Idade } \\
\text { (anos) }\end{array}$ & $\begin{array}{c}\text { Distância } \\
\text { T}_{13}-L_{3} \\
\end{array}$ & $\begin{array}{c}\text { Volume } \\
\text { injetado }(\mathrm{ml}) \\
\end{array}$ & $\begin{array}{c}\text { Volume por } \\
\mathrm{cm}(\mathrm{ml})\end{array}$ \\
\hline 1 & SRD* & 20,1 & 5 & 8.2 & 0.7 & 0.085 \\
\hline 2 & Labrador & 28,0 & 3 & 11.0 & 1.2 & 0.109 \\
\hline 3 & SRD & 28,0 & 10 & 11.0 & 0.9 & 0.081 \\
\hline 4 & SRD & 20,1 & 4.5 & 10.5 & 0.8 & 0.076 \\
\hline 5 & Pastor Alemão & 27,0 & 10 & 12.0 & 1.2 & 0.100 \\
\hline 6 & SRD & 32,0 & 7 & 12.3 & 1.2 & 0.097 \\
\hline 7 & Rottweiler & 31,0 & 6 & 11.0 & 0.8 & 0.072 \\
\hline 8 & Pastor Alemão & 40,0 & 10 & 13.5 & 0.8 & 0.059 \\
\hline 9 & SRD & 25,0 & 11 & 12.5 & 0.8 & 0.064 \\
\hline 10 & Rottweiler & 35,0 & 9 & 12.0 & 1.2 & 0.100 \\
\hline $\begin{array}{l}\text { Média e } \\
\text { desvio } \\
\text { padrão }\end{array}$ & ------ & $\begin{array}{c}28,62 \\
\pm \\
6,22697\end{array}$ & $\begin{array}{c}7,55 \\
\pm \\
2,81316\end{array}$ & $\begin{array}{c}11,4 \\
\pm \\
1.44068\end{array}$ & $\begin{array}{c}0,9 \\
\pm \\
0.21187\end{array}$ & $\begin{array}{r}0.084 \\
\pm \\
0.01683\end{array}$ \\
\hline
\end{tabular}

*SRD: sem raça definida

Um animal cujo peso $(75 \mathrm{~kg})$ e tamanho diferiu dos demais foi excluído do estudo devido ao volume necessário para preenchimento do espaço epidural quase duplicar, sugerindo que à medida que o tamanho corporal aumenta, também se amplia a relação canal vertebral/medula espinal, como já observado por Lecouter e Child (1997), Brisson (2010) e Marioni-Henry (2010). Os valores dos outros cães oscilando entre 0,076 e $0,109 \mathrm{~mL}$ são o dobro ou mais aos encontrados nos segmentos vertebrais T9-L1 (7,8 a 12,3 cm) das colunas vertebrais de cães sem raça definida com peso oscilando entre 10 e $15 \mathrm{~kg}(0,042 \mathrm{~mL}$ por $\mathrm{cm}$ ) (Fernandes, 2016).

Ressalta-se que o contraste de sulfato de bário utilizado pela via epidural permitiu em todas as colunas dos cães confirmar os resultados anteriormente obtidos com o corante Giemsa, corroborando sua utilidade para este tipo de medição, apesar que esta solução de bário possuía densidade visivelmente superior aos dos anestésicos locais usados normalmente para esta via. Acha se também interessante destacar que após coleta do segmento vertebral não se realizou nova secção intervertebral padronizando o forame vertebral de $\mathrm{L}_{5}$ para introduzir o cateter, pois esta nova secção espinal, poderia alterar a relação medula espinal-canal vertebral.

Diante dos resultados alcançados com esta pesquisa, o volume de solução de anestésico que deveria se aplicar por $\mathrm{cm}$ de coluna vertebral entre $\mathrm{T}_{13}$ e $\mathrm{L}_{3}$ de cães com peso entre 20 e $40 \mathrm{Kg}$, oscilaria entre 0,059 e $0,109 \mathrm{~mL}$, com média e desvio padrão de $0,084 \pm 0,01683 \mathrm{~mL}$.

\section{Conflito de Interesse}

Os autores declaram não existir conflito de interesse.

\section{Comitê de Ética}

O projeto de pesquisa foi aprovado pelo Comitê de Ética da Universidade Federal Rural de Pernambuco (UFRPE), sob o número 0001/2014.

\section{Agradecimentos}

Setor de Radiologia do DMV/UFRPE.

\section{Referências}

Almeida, T.F.; Fantoni, D.T.; Mastrocinque, S.; Tatarunas, A.C.; Imagawa, V.H. Epidural anestesia with bupivacaine, bupivacaine and fentanyl, or bupivacaine and sufentanil during intravenous administration of propofol for ovariohyterectomy in dogs. Journal American Veterinary Medical Association, 230: 45-51, 2007.

Brisson, B.A. Intervertebral disc disease in dog. Veterinary Clinics of North America, Small Animal Practice, 40(5): 829-858, 2010. 
Carvalho, Y.K; Luna, S.P.L. Anestesia e Analgesia por via Epidural em cães atualização farmacológica para uma técnica tradicional. Clínica Veterinária, 12(70): 6876, 2007.

Collier, C.B. The intradural space: the fourth place to go astray during epidural block. International Journal of Obstetric Anesthesia, 19(2): 133-141, 2010.

Fernandes, T.H.T. Realização de bloqueio anestésico espinal toracolombar, com cateter epidural em neurocirurgias de cães da raça dachshund. 2016. 78 f. Tese (Doutorado) Programa de Pós-Graduação em Ciência Veterinária. Departamento de Medicina Veterinária, Universidade Federal Rural de Pernambuco, Recife, Pernambuco, 2016.

Franco, A.; Diz, J. The history of the epidural block. Current Anaesthesia \& Critical Care, 11(5), 271-276, 2000.

Freire, C.D. Avaliação da dispersão da bupivacaína na anestesia peridural em cães. Anaesthesia and Intensive Care Medicine, 7(11): 35, 2011.

Jones, R.S. Epidural analgesia in the dog and cat. Veterinary Journal, 161 (2): 123-131, 2001.

Lecouter, A.; Child, G. Afecções da medula espinal. In: Ettinger, S.J.; Feldman, E.C. Tratado de medicina interna veterinária. $4^{\mathrm{a}}$ ed. v.1. São Paulo: Manole, 1997. p.892-977.

Futema, F. Técnicas de anestesia local. In: F antoni, D.T.; Cortopassi, S.R.G. Anestesia em cães e gatos. São Paulo: Roca, 2010. p. 310332.

Lumb, W.V; Jones, E.W. Veterinary anesthesia. Philadelphia. Lea \& Febiger, 1973. 687 p.

Marioni-Henry, K. Feline spinal cord diseases. Veterinary Clinical Small Animal, 40 (5): 1011-1028, 2010.

Massone, F. Anestesia veterinária: farmacologia e técnicas. $4^{\mathrm{a}}$ ed. Rio de Janeiro: Guanabara Koogan, 2003. 225 p.

Otero, P.E. Anestesia locorregional do neuroeixo. In: Klaumann, P. R.; Otero, P. E. Anestesia locorregional em pequenos animais. São Paulo, Editora Roca, 2013. p. 135-176.

Otero, P.E; Fuensalida, S. E; Portela, D. A. Abordagem ao espaço epidural. In: Otero, P.E. Portela, D.A. Manual de anestesia regional em animais de estimação - anatomia para bloqueios guiados por ultrassonografia e neuroestimulação. São Paulo: Med Vet, 2018. p. 316-318.

Skarda, R.T.; Tranquilli, W.J. Técnicas de anestesia e analgesia local e regional: equinos. In: Tranquilli, W.J; Thurmon, J.C.; Grimm, K.A. Lumb \& Jones Anestesiologia e Analgesia veterinária. $4^{\mathrm{a}}$ ed. São Paulo: Roca, 2013. p.660-700.

Vilela, L.M.; Tudury, E.A. Toracotomia: Procedimentos Pré, Trans e Pós-Operatórios. In: Tudury, E.A; Potier, G.M.A. Tratado de técnica cirúrgica veterinária. São Paulo: Med Vet, 2009. p. 263-286. 